\title{
Mobility Aware Users Substantially Boost Spectral Efficiency of Cellular OFDMA Systems
}

\author{
Rainer Schoenen $^{1,2}$, Halim Yanikomeroglu ${ }^{2}$, Bernhard Walke ${ }^{1}$ \\ ${ }^{1}$ Communication Networks (ComNets), Faculty 6, RWTH Aachen University, Germany \\ ${ }^{2}$ Department of Systems and Computer Engineering, Carleton University, Canada
}

\begin{abstract}
Wireless cellular networks perform with a system spectral efficiency which depends on the user terminal distribution over the cell area. Due to an adaptive modulation and coding scheme which depends on the signal-to-interference+noise (SINR) ratio, the achievable data rate is typically an order of magnitude higher in the cell center compared to the cell edge. The performance of IMT-Advanced cellular radio systems like IEEE 802.16m and 3GPP LTE-A will strongly depend on algorithms to cope with the low SINR in the service area. The new paradigm introduced in this paper motivates users to opportunistically change location according to operator recommendation displayed on the user terminal to achieve a much better SINR than currently available. Benefits are the increase of network capacity and higher data rates or potentially a financial incentive for the convinced users. Numeric results based on analysis of IMT scenarios are provided suggesting large cell spectral efficiency gains.
\end{abstract}

Index Terms-IMT-Advanced, Spectral Efficiency, Mobility, Relays, User-in-the-loop

\section{INTRODUCTION}

Recently the IMT-Advanced system performance evaluation has been finalized [1]. The requirements by the ITU [2] are ambitious so that various advanced techniques must be considered, e.g., MIMO, CoMP, and fractional frequency reuse. Multihop techniques (using decode-and-forward relay nodes, $\mathrm{RN}$ ) have also been standardized to either increase cell edge capacity, coverage, or both [3], [4].

The system cell spectral efficiency is the stationary achievable rate averaged in time and over the whole cell area and normalized by the system bandwidth. This corresponds to a scheduler in the base station (BS) which distributes resources fairly among competing user terminals (UTs). When the goal is to fairly give each UT the same data rate, the averaging must be calculated differently [5]. In this case a UT at the cell edge consumes many times the resources compared to a UT in the cell center. The factor, denoted here as $F$, is given by the ratio of the highest to lowest spectral efficiency $\gamma$ in bit $/ \mathrm{s} / \mathrm{Hz}$ on a resource block determined by the adaptive modulation and coding scheme (AMC), for instance, in Table I, $F=7.5$. Especially the AMC is the reason why the performance depends on the location and distribution of user terminals in the cell. This factor becomes even worse if MIMO and advanced receiver algorithms are used.

Operators market their services as if it has, ubiqitiously, the same QoS everywhere in the service area. While this would be desirable, in reality large QoS variance is observed depending on the location. An operator has to provide $F$ times the resources to cell edge users compared to cell center users to provide fair capacity share.

For the voice service $F$ has to be accounted for when provisioning resources for the busy hour. Further, the maximum number of simultaneous phone calls depends on $F$.

For data services (elastic services, best effort, downloads, websurfing), the goal of serving each UT with the same rate (rate-proportional fair service) has the same cost and dependency on $F$ as above. At this point it would be more reasonable to provide each UT a fair amount of resource blocks (resource-proportional fair service). This is the default in IEEE 802.11 like systems anyway. The result is indeed a higher spectral efficiency. The dilemma between throughput capacity and fairness is known quite well [4].

In this paper we propose a new approach to substantially increase the spectral efficiency without changing the physical layer. It begins with the awareness of the user, that the cellular performance depends on the location. Currently users are aware about this fact only in IEEE 802.11 hotspot scenarios. Second, an incentive is needed to improve SINR by moving the UT to another location. Third, the new location must be convenient to reach (e.g., on foot) or the incentive must be enough to make a move. Fourth, there must be an informationassisted guidance on the UTs, showing directions or even a map of the area (Fig. 1). This operator database is filled by all UTs over all times, so it is very substantial. As a result, some users would be motivated to move to a location with better SINR and, accordingly, will contribute with a factor of typically two to four (up to $F$ ) to the increase of the system's spectral efficiency. The proposal is opportunistic, not mandatory, and users do not need to comply, for example when driving in a car.

The method is studied with analytic and numeric tools on the example of the ITU-R IMT-Advanced standard test scenarios. Our results show that a substantial gain in cell spectral efficiency can be achieved with some reasonable effort required by the user.

TABLE I

PHY MODES AND SINR INTERVALS

\begin{tabular}{|c|c|c|c|c|c|c|c|c|}
\hline Index $m$ & 1 & 2 & 3 & 4 & 5 & 6 & 7 & 8 \\
\hline SINR $\sigma[\mathrm{dB}]$ & 0.9 & 2.1 & 3.8 & 7.7 & 9.8 & 12.6 & 15.0 & 18.2 \\
\hline Modulation & \multicolumn{5}{|c|}{ QPSK } & \multicolumn{5}{c|}{16 -QAM } & \multicolumn{2}{c|}{ 64-QAM } \\
\hline Coding rate & $1 / 3$ & $1 / 2$ & $2 / 3$ & $1 / 2$ & $2 / 3$ & $5 / 6$ & $2 / 3$ & $5 / 6$ \\
\hline$\gamma[\mathrm{b} / \mathrm{s} / \mathrm{Hz}]$ & $2 / 3$ & 1 & $4 / 3$ & 2 & $8 / 3$ & $10 / 3$ & 4 & 5 \\
\hline
\end{tabular}




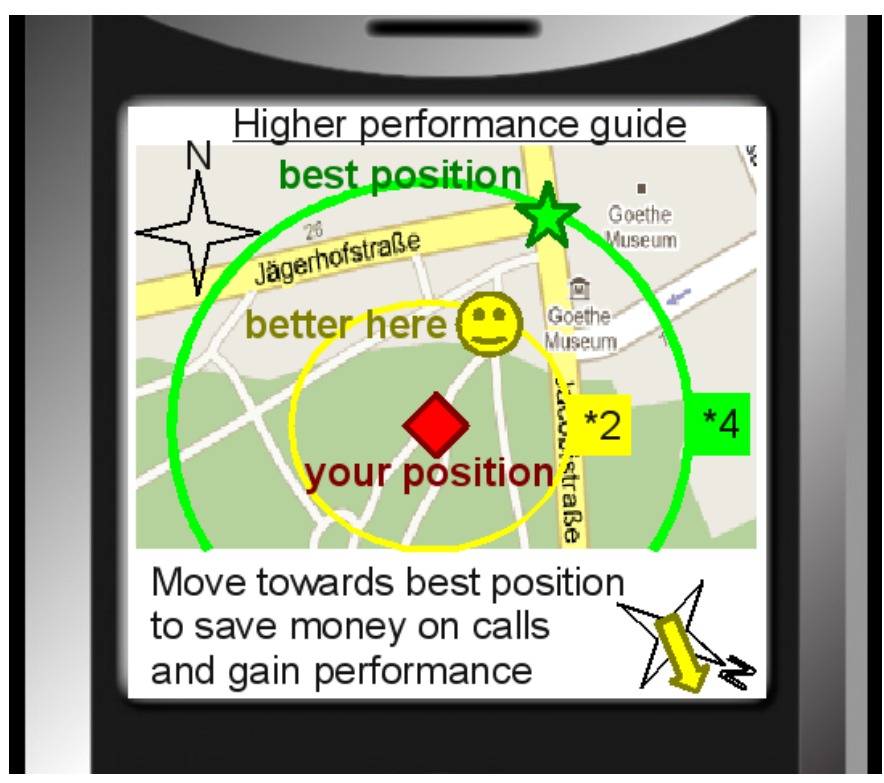

Fig. 1. Exemplary UT display showing distance, direction and benefit. If this is attractive, the user moves to a location with higher spectral efficiency (with probability $p_{M}$ ).

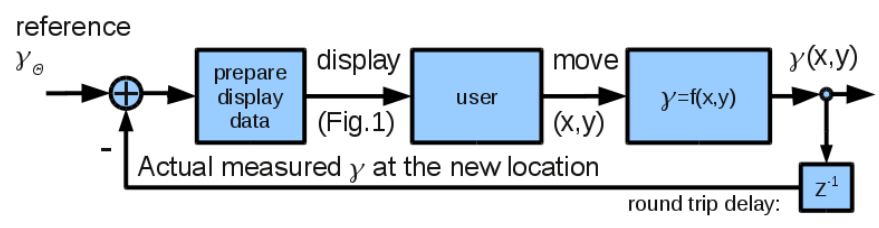

Fig. 2. Closed loop with the user as the system to control.

\section{SySTEM MODEL}

In this paper we assume the IMT-Advanced system model [2]. Table II provides the data for the scenarios taken into account. They are representative for the whole range between densely populated (UMi) to countryside setups (RMa). Table III specifies parameters related to the LTE-Advanced radios. The pathloss is calculated using the two parallel models (with/without line-of-sight) and distance-dependent probability $p_{L O S}(r)$ to select which one is used [6], [7]. Shadowing is not modeled here. In the presence of shadowing returns are expected to be even more favorable. A single antenna setup is assumed, as the main implications are not affected by the presence or absence of MIMO. In a multicellular context with reuse-1 interference is the major limitation. At the cell borders $S I N R$ is close to zero with high fluctuations. The system model includes optional relay nodes (RN) as well (0 or $3 \mathrm{RN}$ per cell). It is important to select the serving station (BS or RN) by the least resources decision, i.e., the decision of taking the single or multihop route is taken by considering which option uses less resources, not by choosing $\max (\mathrm{SINR})$. Over the cell area SINR $(\sigma)$ results are obtained by numeric analysis and are translated to spectral efficieny $\gamma$ in bit/s/Hz [8] according to Table I and the modulation and coding performance results [9].
TABLE II

IMT-ADVANCED SCENARIO SPECIFICATIONS

\begin{tabular}{|c|c|c|c|c|}
\hline Scenario & $\begin{array}{c}\text { Urban } \\
\text { micro } \\
\text { UMi }\end{array}$ & $\begin{array}{c}\text { Urban } \\
\text { macro } \\
\text { UMa }\end{array}$ & $\begin{array}{c}\text { Suburban } \\
\text { macro } \\
\text { SMa }\end{array}$ & $\begin{array}{c}\text { Rural } \\
\text { macro } \\
\text { RMa }\end{array}$ \\
\hline Inter-BS distance & $200 \mathrm{~m}$ & $500 \mathrm{~m}$ & $1299 \mathrm{~m}$ & $1732 \mathrm{~m}$ \\
\hline BS height & $10 \mathrm{~m}$ & $25 \mathrm{~m}$ & $35 \mathrm{~m}$ & $35 \mathrm{~m}$ \\
\hline Antenna tilt & $-12^{\circ}$ & $-12^{\circ}$ & $-6^{\circ}$ & $-6^{\circ}$ \\
\hline$f_{C}$ & $2.5 \mathrm{GHz}$ & $2.0 \mathrm{GHz}$ & $2.0 \mathrm{GHz}$ & $0.8 \mathrm{GHz}$ \\
\hline Tx power & $44 \mathrm{dBm}$ & $49 \mathrm{dBm}$ & $49 \mathrm{dBm}$ & $49 \mathrm{dBm}$ \\
\hline
\end{tabular}

TABLE III

TECHNOLOGY PARAMETERS ACCORDING TO LTE-A

\begin{tabular}{|c|c|}
\hline Bandwidth (FDD) & $20 \mathrm{MHz}$ DL \\
\hline Traffic & full load; best effort \\
\hline Antenna gain (boresight) & $17 \mathrm{dBi}$ \\
\hline Sectors/cell & 3 \\
\hline Antenna aperture horizontal $\theta_{3 d B}$ & $70^{\circ}$ \\
\hline Antenna aperture vertical $\phi_{3 d B}$ & $15^{\circ}$ \\
\hline Thermal noise & $-174 \mathrm{dBm} / \mathrm{Hz}$ \\
\hline UT noise figure & $5 \mathrm{~dB}$ \\
\hline
\end{tabular}

\section{USER IN THE LOOP}

The new concept provides suitable information to the user, e.g., as shown in Figure 1, and the user should be convinced to change his location voluntarily from his current location $\overrightarrow{p_{1}}=\left(x_{1}, y_{1}\right)$ to $\overrightarrow{p_{2}}$. Thus the user becomes part of a control loop (Figure 2). System theory including human elements is inspired by [10]; power supply companies have been trying out such approaches [11] in recent years. The network controller knows the current signal quality $\sigma\left(\overrightarrow{p_{1}}\right)$ (SINR-based) or $\gamma\left(\overrightarrow{p_{1}}\right)$ from UT measurements, and the expected level $\gamma\left(\overrightarrow{p_{2}}\right)$ from a database of measurements of all UTs at all locations in the past. The user knows his utility advantage of $\Delta u_{1,2}=$ $u\left(\overrightarrow{p_{2}}\right)-u\left(\overrightarrow{p_{1}}\right)$ when doing the move. This utility $u$ can be either financial (savings for voice during busy hours) or an increased data rate (for best effort data traffic). The network provides the information in which direction or to which location to move by the gradient $-\nabla \sigma(\vec{p})$ of the potential field at position $\overrightarrow{p_{1}}$. The user should have all information to make his decision. UT devices would ideally have GPS onboard, but the network can still support ranging by BS-based triangulation and give hints for movement. The user can see in which direction to move best and how far $d_{1,2}=\left|\overrightarrow{p_{2}}-\overrightarrow{p_{1}}\right|$ the next improvement step is.It is assumed that a fraction $p_{M}$ of users actually participates in moving, the rest stay at place. $p_{M}$ accounts for users that cannot move, do not want to move, or have no sufficient incentive to move. The output of the user block (Fig. 2) is the new location $\overrightarrow{p_{2}}$. It is described by a Bernoulli random process where $p_{M}$ is the probability of a move from $\overrightarrow{p_{1}}$ to $\overrightarrow{p_{2}}$ for $d_{1,2}$ meters and $\left(1-p_{M}\right)$ of no movement at all. The target value $\gamma_{\Theta}$ is the least $\gamma$ this UT should achieve after the movement.

\section{Performance Results}

In this paper the statistics of the movement distance and the resulting spectral efficiency are determined by numeric analysis of the IMT-Advanced scenarios.Both increases of $\Delta \gamma$ and $d$ are weighted by $p_{M}$, because $\left(1-p_{M}\right)$ of the users are 


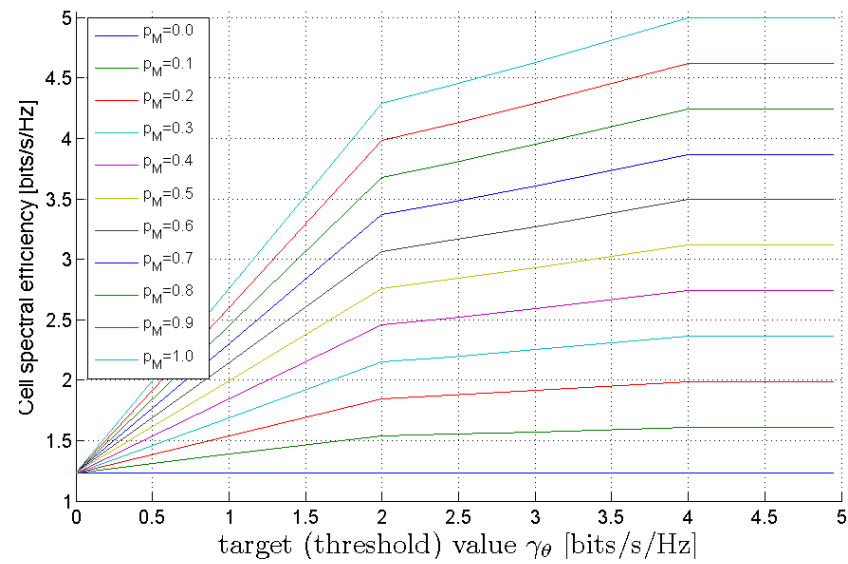

Fig. 3. Observed spectral efficiency $\gamma$ in bit/s/Hz depending on the reference $\gamma_{\Theta}$ in the SMa scenario.

TABLE IV

GRoss SPECTRAL EFFICIENCY RESUlTS FOR THE IMT SCENARIO EVALUATION [BIT/S/Hz/SECTOR] WITH $\gamma_{\Theta}=2.5 \mathrm{BIT} / \mathrm{S} / \mathrm{Hz}$

\begin{tabular}{|c|c|c|c|c|}
\hline Scenario & UMi & UMa & SMa & RMa \\
\hline 0RN,HUD & 1.567 & 1.254 & 1.234 & 1.974 \\
\hline 0RN,UIL, & 2.170 & 1.995 & 2.836 & 2.509 \\
$p_{M}=0.5 \rightarrow \bar{d}=$ & $4.4 \mathrm{~m}$ & $4.7 \mathrm{~m}$ & $7.8 \mathrm{~m}$ & $30.7 \mathrm{~m}$ \\
\hline 0RN,UIL, & 2.772 & 2.735 & 4.437 & 3.045 \\
$p_{M}=1 \rightarrow \bar{d}=$ & $8.8 \mathrm{~m}$ & $9.4 \mathrm{~m}$ & $15.6 \mathrm{~m}$ & $61.4 \mathrm{~m}$ \\
\hline 3RN,HUD & 1.945 & 1.804 & 1.825 & 2.310 \\
\hline 3RN,UIL, & 2.333 & 2.239 & 2.858 & 2.654 \\
$p_{M}=0.5 \rightarrow \bar{d}=$ & $1.9 \mathrm{~m}$ & $1.7 \mathrm{~m}$ & $5.0 \mathrm{~m}$ & $12.0 \mathrm{~m}$ \\
\hline 3RN,UIL, & 2.721 & 2.674 & 3.892 & 2.998 \\
$p_{M}=1 \rightarrow \bar{d}=$ & $3.7 \mathrm{~m}$ & $3.4 \mathrm{~m}$ & $10.0 \mathrm{~m}$ & $23.9 \mathrm{~m}$ \\
\hline
\end{tabular}

assumed to be not willing to move. Users who do not need to move as they are already at good positions are accounted with $d=0$. Rather moderate values for the parameters have been chosen in order to be realistic: $p_{M}=\frac{1}{2}$ and $\gamma_{\Theta}=2.5 \mathrm{bit} / \mathrm{s} / \mathrm{Hz}$. The scenarios were investigated with and without relays (RN).

The spectral efficiency and average distance results $\bar{d}$ for the IMT-Advanced scenarios are provided in Table IV, where HUD means homogeneous user distribution (classic conservative model without movement [7]) and UIL means user-in-theloop (progressive model) with anisotropic, nonhomogeneous user density. The total spectral efficiency is increased by $25 \%$ to more than $100 \%$, depending on the IMT scenario, even with moderate parameters. This increase requires an effective movement of just a few meters on average. The parameter $p_{M}$ influences the result linearly, whereas $\gamma_{\Theta}$ does not (Figure 3).

Figure 4 shows the cumulative density function (CDF) of the required movement. It explains how many users have to move by how far. In all scenarios (except RMa) $80 \%$ to $90 \%$ of the users need to move less than $10 \mathrm{~m}$. Relays reduce the effort to move for the user due to the shorter distance to the closest RN. The analysis results suggest that the proposed approach works well, because the movement effort is low, while the gains are high. Results for other scenarios can be found in [12].

\section{CONCLUSION}

This paper introduced a novel approach to increase the system spectral efficiency by the help of participating users. It is not that these users are interested in this goal itself [13], but

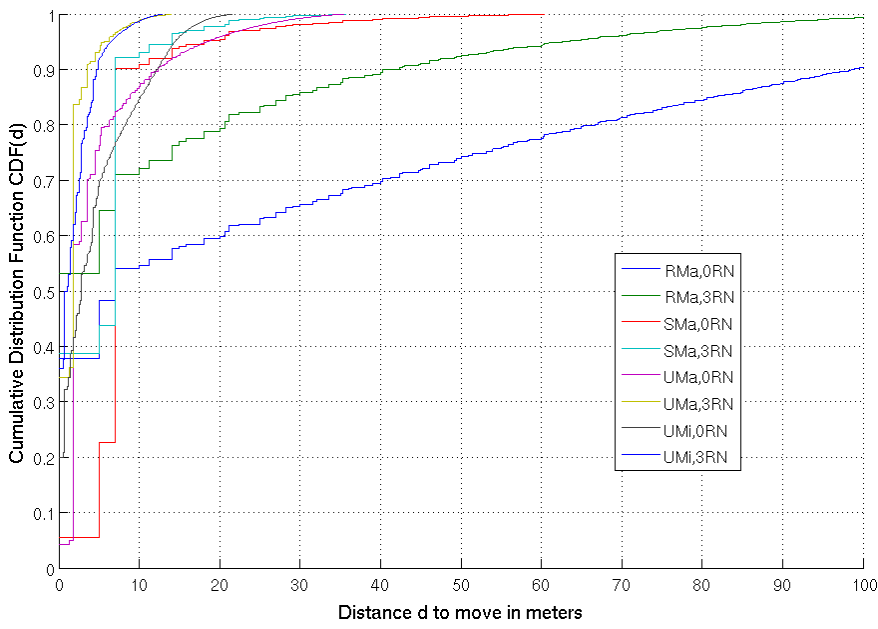

Fig. 4. CDF of the movement distance $d$ for all IMT scenarios. 0 and 3 in the scenario name parameter mean the number of relay nodes in the cell.

they have an incentive to voluntarily improve their application data rate or reduce the cost of connections during busy hours by performing a voluntary movement to a location of higher performance. Studies using the IMT-Advanced evaluation scenarios showed substantial gains up to $200 \%$, depending on the percentage of users involved. The distances to move are in the order of a few meters in most cases. It is recommended the user-in-the-control-loop techniques should be investigated further, as this seems to be promising to tackle many economic and ecologic problems.

\section{REFERENCES}

[1] J. Monserrat et al., "Advanced radio resource management for IMTAdvanced in WINNER+ (II)," in Proc. ICT-MobileSummit June 2010.

[2] ITU, "Report ITU-R M2135; Guidelines for evaluation of radio interference technologies for IMT-Adcanced", 2008.

[3] R. Pabst, B. Walke, D. C. Schultz, H. Yanikomeroglu et al., "Relaybased deployment concepts for wireless and mobile broadband radio," IEEE Communications Magazine, pp. 80-89, Sep 2004.

[4] M. Salem, H. Yanikomeroglu, D. Falconer et al., "An overview of radio resource management in relay-enhanced OFDMA-based networks," IEEE Communications Surveys and Tutorials, vol. 12, no. 3, 2010.

[5] C. Hoymann and S. Goebbels, "Dimensioning cellular wimax part I: Singlehop networks," in Proc. European Wireless 2007, Paris, France, Apr 2007, p. 7.

[6] D. Bültmann, T. Andre, and R. Schoenen, "Analysis of 3GPP LTEAdvanced cell spectral efficiency," in Proc. PIMRC 2010, Istanbul, Turkey, September 2010.

[7] R. Schoenen and C. Teijeiro, "System level performance evaluation of LTE with MIMO and relays in reuse-1 IMT-Advanced scenarios," in Proceedings of the IEEE WiCom, Chengdu, China, Sep 2010.

[8] K. Brueninghaus and D. e. a. Astely, "Link performance models for system level simulations of broadband radio access systems," in Proc. PIMRC'2005, Berlin, Sep 2005, pp. 2306-2311.

[9] R. Schoenen and B. Walke, "On PHY and MAC performance of 3GLTE in a multi-hop cellular environment," in Proc. IEEE WiCom 2007, Shanghai, China, Sep 2007.

[10] R. Zimmerman, Das System Mensch. Artech House, 2003, ISBN 300-012784-4.

[11] A. Faruqui and R. Earle, "Demand response and advanced metering," Regulation, vol. 29, no. 1, pp. 24-27, 2006.

[12] R. Schoenen, "On increasing the spectral efficiency more than $100 \%$ by user-in-the-control-loop," in Proc. of the 16th Asia-Pacific Conference on Communications (APCC), Auckland, October 2010.

[13] G. Hardin, "The tragedy of the commons," Science, vol. 20, pp. 124347, 1968. 